\title{
OPEN Functional and histologic imaging of urinary bladder wall after exposure to psychological stress and protamine sulfate
}

\author{
Tetsuichi Saito ${ }^{1,4,6}$, T. Kevin Hitchens ${ }^{2,3}$, Lesley M. Foley ${ }^{2}$, Nishant Singh ${ }^{1,6}$, \\ Shinsuke Mizoguchi ${ }^{1}$, Masahiro Kurobe ${ }^{1}$, Daisuke Gotoh ${ }^{1}$, Teruyuki Ogawa ${ }^{4}$, \\ Tomonori Minagawa ${ }^{4}$, Osamu Ishizuka ${ }^{4}$, Christopher Chermansky ${ }^{1}$, Jonathan Kaufman ${ }^{5}$, \\ Naoki Yoshimura ${ }^{1} \&$ Pradeep Tyagi $^{1 \bowtie}$
}

To quantify the urinary bladder wall $\mathrm{T}_{1}$ relaxation time $\left(\mathrm{T}_{1}\right)$ before and after the instillation contrast mixture in rats previously subjected to water avoidance stress (WAS) and/or acute exposure to protamine sulfate (PS). Female Wistar rats were randomized to receive either sham (control) or $1 \mathrm{~h}$ of WAS for ten consecutive days before the evaluation of nocturnal urination pattern in metabolic cages. $T_{1}$ mapping of urinary bladder wall at $9.4 \mathrm{~T}$ was performed pre- and post- instillation of $4 \mathrm{mM}$ Gadobutrol in a mixture with $5 \mathrm{mM}$ Ferumoxytol. Subsequently, either $\mathrm{T}_{1}$ mapping was repeated after brief intravesical PS exposure or the animals were sacrificed for histology and analyzing the mucosal levels of mRNA. Compared to the control group, WAS exposure decreased the single void urine volume and shortened the post-contrast $\mathrm{T}_{1}$ relaxation time of mucosa- used to compute relatively higher ingress of instilled Gadobutrol. Compromised permeability in WAS group was corroborated by the urothelial denudation, edema and ZO-1 downregulation. PS exposure doubled the baseline ingress of Gadobutrol in both groups. These findings confirm that psychological stress compromises the paracellular permeability of bladder mucosa and its non-invasive assay with MRI was validated by PS exposure.

Bladder Pain Syndrome/Interstitial Cystitis (BPS/IC) is a debilitating condition associated with severe bladder pain and lower urinary tract symptoms (LUTS). Importantly, disease history of patients afflicted with LUTS, functional pain disorders, and $\mathrm{BPS} / \mathrm{IC}^{1-5}$ tends to show a prior or recent exposure to psychological stress, but whether stress is coincident, or a causative factor of BPS/IC is yet to be investigated.

The epidemiologic link between the psychological stress and the BPS/IC symptoms of urinary bladder pain and increased urinary frequency have received mechanistic support from the evidence of visceral hyperalgesia in rodent models of psychological stress ${ }^{6-9}$. Moreover, a structurally deficient urothelium of BPS/IC patients with the proliferation of mast cells $s^{4,5,10-12}$ being mirrored in the rodent models of chronic water avoidance stress (WAS) ${ }^{13,14}$ supports their validity as a rodent model for BPS/IC. However, the mechanistic link between psychological stress and the BPS/IC symptoms remains to be tested in a radiation-free and in a clinically viable manner ${ }^{15,16}$ and consequently there is an unmet need for a minimally invasive assay of mucosal permeability without the site-selection bias of bladder biops ${ }^{2}$. Such a permeability assay could have many clinical applications including objective phenotyping of the heterogeneity in BPS/IC symptoms for informing the treatment selection and the development of new drugs. Instead of measuring the concentration of instilled polar dyes, i.e., Evans blue or Trypan blue in bladder harvested after animal sacrifice to measure the bladder permeability ${ }^{17-20}$, here we seek to non-invasively measure the bladder permeability in a clinically viable manner by replacing the instillation of polar dyes with the instillation of an extracellular $\mathrm{T}_{1}$ shortening agent, Gadolinium chelate ${ }^{21}$ and then compute the concentration of instilled Gadolinium chelate diffusing into bladder mucosa from the changes in the physical

\footnotetext{
${ }^{1}$ Department of Urology, School of Medicine, University of Pittsburgh, E313 Montefiore Hospital, 3459 Fifth Avenue, Pittsburgh, PA, USA. ${ }^{2}$ Animal Imaging Center, University of Pittsburgh, Pittsburgh, USA. ${ }^{3}$ Department of Neurobiology, University of Pittsburgh, Pittsburgh, USA. ${ }^{4}$ Department of Urology, Shinshu University, Matsumoto, Japan. ${ }^{5}$ Lipella Pharmaceuticals Inc, Pittsburgh, PA, USA. ${ }^{6}$ These authors contributed equally: Tetsuichi Saito and Nishant Singh. ${ }^{\boxplus}$ email: tyagip@upmc.edu
} 
parameter, proton spin-lattice relaxation time ( $\mathrm{T}_{1}$ relaxation time) of mucosa measured via Magnetic Resonance Imaging (MRI), pre and post-instillation of the Gadolinium chelate- Gadobutrol. Gadobutrol being a paramagnetic agent develops a magnetic moment in the magnetic field of scanner and the resulting induction of a local magnetic field shortens the $\mathrm{T}_{1}$ relaxation time of water protons present in tissue to brighten the tissue being imaged. The premise for the proposed translational research is most strongly supported by the successful use of MRI for measuring the blood brain barrier permeability compromised by inflammation in mildly demented patient $^{22}$. Since the volume of distribution for the instilled or injected Gadolinium chelate is restricted to the vascular and extravascular-extracellular space available in a given tissue ${ }^{23}$; edematous changes in bladder wall provoked by WAS ${ }^{13,14}$ are expected to enlarge the physical space for the residence of diffused Gadobutrol and affect the MR images of diseased bladder, accordingly.

Therefore, considering that compromised mucosal permeability alters the biochemical milieu of bladder wall $^{17}$, we hypothesize that the measurement of the physical parameter, $\mathrm{T}_{1}$ relaxation time in milliseconds (ms) for bladder mucosa measured pre and post-instillation of Gadobutrol will serve as an index for mucosal permeability in WAS model. Here, we quantified the $\mathrm{T}_{1}$ relaxation time of bladder wall- the first order rate constant for the exponential rise in signal intensity from a series of $\mathrm{T}_{1}$ weighted spin echo images acquired at Variable Repetition Time (VTR)- a method previously used on human bladder at $1.5 \mathrm{~T}^{24}$. Therefore, instead of a biochemical assay for permeability in BPS/IC patients ${ }^{2,10,16}$, the overall focus of this research study is to empirically demonstrate the utility of contrast enhanced MRI for assaying the mucosal permeability of WAS rat with or without the intravesical exposure to protamine sulfate (PS) - an agent known to increase mucosal permeability of rodent ${ }^{25-27}$ as well as of human bladder ${ }^{16}$.

\section{Methods}

All experiments were conducted on ten-week-old female Wistar rats $(n=21)$ in accordance with the National Institutes of Health Guide for the Care and Use of Laboratory Animals and ARRIVE guidelines and approved by the University of Pittsburgh Institutional Animal Care and Use Committee.

Water Avoidance Stress (WAS) model. Animals were randomly divided into control $(\mathrm{N}=9)$ or WAS $(\mathrm{N}=12)$ groups. Animals underwent 1 -h exposure to WAS or control setting for 10 consecutive days as published previously ${ }^{28}$. Briefly, rats from both groups were placed on a glass platform $(8 \times 8 \mathrm{~cm})$ in the middle of a plastic container, $90 \mathrm{~cm}$ in diameter and $50 \mathrm{~cm}$ in height, which was left empty for the control group and filled with $49 \mathrm{~cm}$ of water for the WAS group. Since psychological stress is known to modulate colonic motility, number of fecal pellets excreted after WAS were measured.

Metabolic cage. After 10 days of WAS, all rats were placed in a metabolic cage (Tecniplast, Buguggiate VA, Italy) for $12 \mathrm{~h}$ (from $7 \mathrm{pm}$ to $7 \mathrm{am}$ ) with food and water ad libitum. Voiding frequency, single void urine volume and total urine volume were measured.

Voxel-wise $\mathrm{T}_{1}$ mapping. As described recently for mouse bladder ${ }^{29}$, MRI was performed in a $9.4 \mathrm{~T} / 30 \mathrm{~cm}$ Bruker AVANCE III HD scanner (Bruker BioSpin, Billerica, MA, USA) running ParaVision 6.0.1 equipped with a $12 \mathrm{~cm}$ BGA-12SHP gradient set, an $86 \mathrm{~mm}$ transmit coil and $35 \mathrm{~mm} 2 \times 2$ receiver array over the bladder. Rats were maintained under isoflurane anesthesia via a nose cone (2-3\% in 1:1 Oxygen: Air). Following pilot scans, a Rapid Acquisition with Relaxation Enhancement (RARE) sequence with $\mathrm{VTR}^{24}$ was used to generate $\mathrm{T}_{1}$ maps of the bladder wall before and after transurethral $0.3 \mathrm{~mL}$ instillation of an aqueous contrast mixture $(\mathrm{CM})$ containing $4 \mathrm{mM}$ Gadobutrol (Gadavist; Bayer, Wayne, NJ, USA) and $5 \mathrm{mM}$ Ferumoxytol (AMAG Pharmaceuticals Inc., Waltham, MA, USA) via a 24-gauge angiocatheter (Becton-Dickinson Infusion Therapy Systems, Sandy, UT, USA). Imaging parameters were as follows: $\mathrm{TR}=400,842,1,410,2,208,3,554$ and 10,000 ms, echo time (TE) $=7 \mathrm{~ms}$, 9 contiguous $0.7 \mathrm{~mm}$-axial slices, RARE factor $=2,2$ signal averages, $20 \times 20 \mathrm{~mm}$ field of view (FOV) and a matrix size of $200 \times 200$, zero-filled to $400 \times 400$ prior to Fourier transform. $T_{1}$ maps were processed using a 3-parameter single exponential function.

$T_{1}$ measurement after protamine sulfate (PS) exposure for permeability validation. Following post contrast MRI, we first expelled the CM by pressing on the bladder followed by two transurethral irrigation of saline $0.5 \mathrm{~mL}$ prior to the instillation of $0.5 \mathrm{~mL}$ of PS (Sigma-Aldrich, St. Louis, MO, USA) $1 \% \mathrm{w} / \mathrm{v}$ in normal saline. After a dwell time of $30 \mathrm{~min}$, PS was expelled from the bladder for a second instillation of CM $(0.3 \mathrm{~mL})$ for a repeat post-contrast $\mathrm{T}_{1}$ mapping.

Real-time RT-PCR and histology. WAS and control group rats not exposed to PS were euthanized, the bladder harvested for either isolating RNA or histology after 30 min fixing with $4 \%$ paraformaldehyde (SigmaAldrich, St. Louis, MO, USA) at $4{ }^{\circ} \mathrm{C}$, followed by overnight immersion in $20 \%$ sucrose at $4{ }^{\circ} \mathrm{C}$ for cryoprotection in optimal cutting temperature (OCT) preservation medium (Tissue-Tek, Torrance, CA, USA) before cryosectioning. Sections were stained with Hematoxylin and Eosin (Fisher Healthcare, Pittsburgh, USA) and then digital photographs were acquired. Researchers blinded to the identity of sections examined the regions of interest at low and high magnification for visible damage to the mucosal layer, edema, infiltration of polymorphonuclear inflammatory cells, and vascular congestion. The sections of two groups were subjectively scored as follows: a unit score was assigned for the evidence of mucosal thinning, another unit score for the evidence of vascular congestion in same area and likewise for the infiltration of inflammatory cells. All the scores were added 


\begin{tabular}{|l|l|l|l|l|}
\hline Voiding parameter & Control & WAS & $\boldsymbol{p}$ value & 95\% confidence interval \\
\hline Single Void Volume $(\mathrm{mL})$ & $0.76 \pm 0.11$ & $0.35 \pm 0.04$ & $<0.05$ & -0.6526 to -0.1706 \\
\hline Number of micturition in $12 \mathrm{~h}$ & $13.5 \pm 3.76$ & $15 \pm 3.32$ & $>0.05$ & -10.08 to 7.085 \\
\hline Urine output $(\mathrm{mL})$ & $11.7 \pm 6.25$ & $4.7 \pm 2.24$ & $<0.05$ & -13.79 to -0.06559 \\
\hline Voiding Interval $(\mathrm{min})$ & $58.0 \pm 23.2$ & $60.6 \pm 22.3$ & $>0.05$ & -31.92 to 36.96 \\
\hline
\end{tabular}

Table 1. Metabolic cage urination parameters. All values are expressed as mean \pm SD.

to arrive at a composite score for the inflammation of each section from control and WAS groups when viewed at $40 \times$ magnification.

Mucosa of freshly harvested bladder was separated ${ }^{30,31}$ and frozen immediately at $-80{ }^{\circ} \mathrm{C}$ until isolation of total RNA using TRizol reagent (Invitrogen, Carlsbad, CA, USA) for real-time PCR using primers and cycle conditions as previously reported ${ }^{32}$ for relative quantity of tight junction ZO-1 transcript normalized to GAPDH mRNA. The contamination of lamina propria cannot be excluded by our technique of manual separation of mucosa from detrusor. Primer sequences for ZO-1 (Forward 5'-GCGAGGCATCGTTCCTAATAAG-3'; Reverse 5'-TCGCCACCTGCTGTCTTTG-3' and GAPDH forward primer 5'-AGACAGCCGCATCTTCTTGT-3'; Reverse 5'-GATACGGCCAAATCCGTTC-3' were procured from Integrated DNA technologies, Coralville, IA, USA.

Statistical analysis. All values are expressed as mean \pm SD. Since $\mathrm{T}_{1}$ relaxation times are normally distributed, two-way analysis of variance (ANOVA) followed by Sidak's multigroup comparison or unpaired $t$ test for pairwise comparison of physiological parameters and Mann-Whitney test for the semi-quantitative parameter of inflammation scores using GraphPad Prism ver 8.0.0 (GraphPad Software, San Diego, CA, USA). Values at $p<0.05$ were considered significant.

\section{Results}

Colonic motility index of psychological stress. When exposed to daily WAS for an hour over 10 days, rats in the WAS group expelled a significantly higher number of fecal pellets $(4.54 \pm 0.29$ pellets/hour vs $1.08 \pm 0.11$ pellets/hour, $p<0.0001$ ) compared to the control group.

Metabolic cage. Rats in the WAS group voided significantly smaller single volumes in nocturnal void of $0.35 \pm 0.04 \mathrm{~mL}$ compared to $0.76 \pm 0.11 \mathrm{~mL}$ in the control group $(p<0.05)$ without any significant difference in the micturitions recorded over $12 \mathrm{~h}$ time-frame $(15 \pm 3.32 / 12 \mathrm{~h}$ vs $13.5 \pm 3.76 / 12 \mathrm{~h})$. Total urine volume was also significantly decreased in WAS rats $(11.7 \pm 6.25 \mathrm{~mL}$ vs $4.7 \pm 2.24 \mathrm{~mL} ; \mathrm{p}<0.05)$ (Table 1$)$.

Voxel-wise $\mathrm{T}_{1}$ mapping. First a series of raw $\mathrm{T}_{1}$ weighted images were acquired at $\mathrm{TR}$ of $400,842,1410$, 2208, 3554 and 10,000 ms while keeping TE constant at $7 \mathrm{~ms}$ to reduce the component of $\mathrm{T}_{2}$ weighting in the acquired images. For illustration, raw $\mathrm{T}_{1}$ weighted image taken at $\mathrm{TR}$ of $1410 \mathrm{~ms}$ is shown for each group in each experimental setting in Fig. 1A-F, which were acquired before the instillation of CM (pre-contrast), after $0.3 \mathrm{~mL}$ instillation of CM but prior to the exposure to protamine sulfate PS (post-contrast) and after PS exposure(postprotamine). In order to reconstruct the geographical representation of true $\mathrm{T}_{1}$ for different bladder wall layers within the FOV of $20 \times 20 \mathrm{~mm}$-voxel-wise $\mathrm{T}_{1}$ mapping - $\mathrm{T}_{1}$ was calculated from nonlinear least square data fitting of TR dependent signal intensity increase in every voxel of multiple raw $\mathrm{T}_{1}$ weighted images acquired at different TR of 400, 842, 1410, 2208, 3554 and 10,000 ms. The color-coded $\mathrm{T}_{1}$ maps (Fig. 1Ai-Fii) display purple and red color for $\mathrm{T}_{1}$ of 0 and $7000 \mathrm{~ms}$, respectively.

To avoid the variability from bladder distension during imaging, urine production by the animals was reduced with $12 \mathrm{~h}$ water restriction prior to imaging and the representative $\mathrm{T}_{1}$ weighted images for the control (Fig. 1A-C) and WAS group (Fig. 1D-F) displays that the bladder distension was not variable across groups.

We compared the pre and post contrast $\mathrm{T}_{1}$ for 20 pixels in an ROI of the bladder wall highlighted by the square boxes in panels Ai-Fi-magnified in the respective lower panels (Aii-Fii) to visualize the sandwich of lamina propria (L) between the mucosa (U) and detrusor (D) layers. Given the pixel size for display is $50 \mu \mathrm{m}$ in Fig. 1, at least two pixels display individual cells ${ }^{33}$ of the luminal cell layer of mucosa. The pre-contrast $\mathrm{T}_{1}$ values for the U ( $3509 \pm 359 \mathrm{~ms}$ vs $3460 \pm 280 \mathrm{~ms}$; Fig. $2 \mathrm{~B})$, for L ( $3176 \pm 144 \mathrm{~ms}$ vs $3058 \pm 274 \mathrm{~ms})$ and D $(2632 \pm 175 \mathrm{~ms}$ vs $2743 \pm 84 \mathrm{~ms}$ ) layers of control and WAS groups respectively, were comparable (Fig. 1Aii,Dii). After the instillation of $\mathrm{CM}$ in lumen, the long $\mathrm{T}_{1}$ of mucosa (Fig. 1Aii,Dii) is shortened to $1420 \pm 144 \mathrm{~ms}$ and $965 \pm 111 \mathrm{~ms}$ (Fig. $2 \mathrm{~B}$ ) in post-contrast $\mathrm{T}_{1}$ maps of control and WAS group, respectively (Fig. 1Bii,Eii) and the mean values of each group in different experimental settings were used for calculating the mean values of Gadobutrol permeability in each group.

Gadobutrol permeability. As displayed in Fig. 1Ai-Di, the mucosa of WAS group exhibits significantly shorter post-contrast $\mathrm{T}_{1}$ relaxation time than that of controls (Fig. $2 \mathrm{~B}$ ) and the between group differences were further accentuated upon PS exposure (Fig. $2 \mathrm{~B}$ ). The shortening of $\mathrm{T}_{1}$ relaxation time can help us derive Gadobutrol permeability in any given region of interest (ROI) of bladder wall. Because the longitudinal relaxa- 

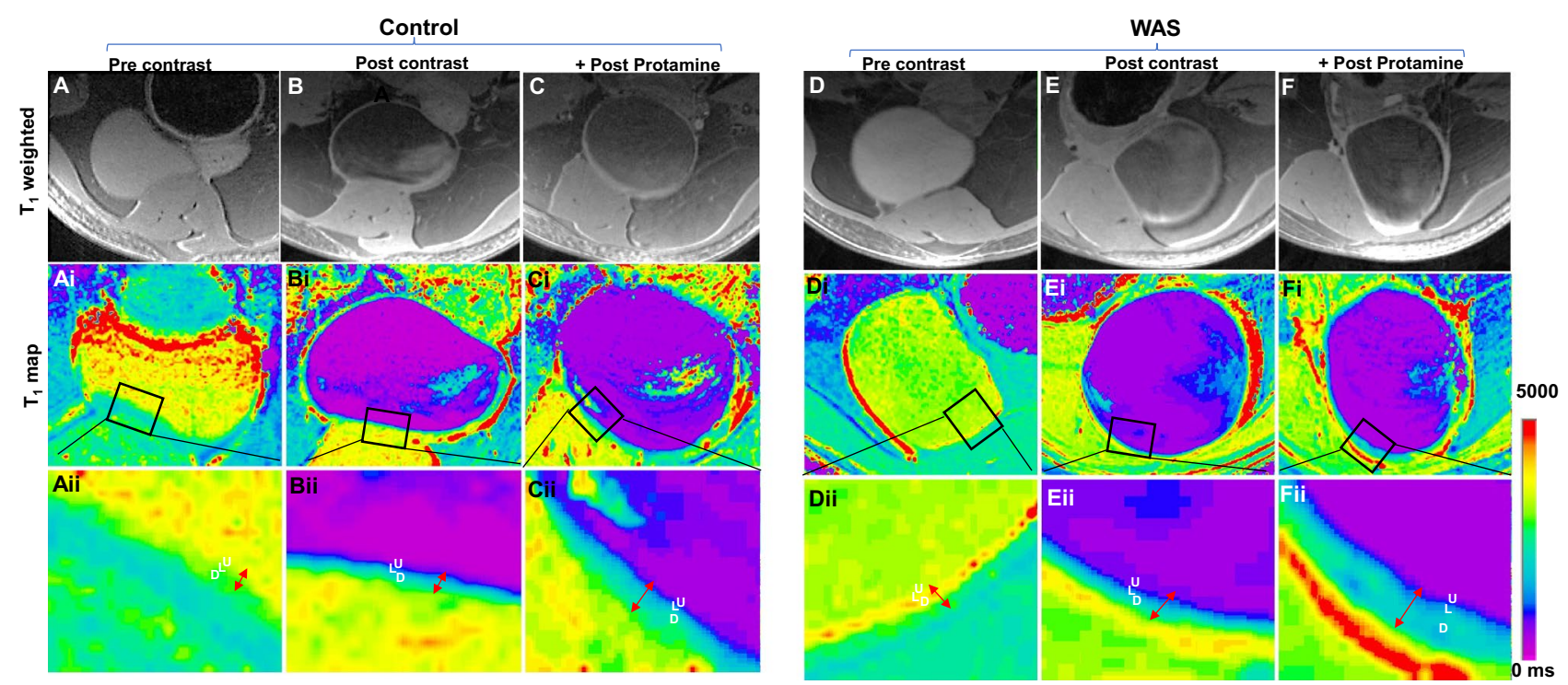

Figure 1. Effect of WAS and PS on voxel-wise $T_{1}$ mapping- $T_{1}$ weighted images (TR/TE 1410/7ms) acquired before the instillation of CM (pre-contrast), after the instillation of CM but prior to the exposure to protamine sulfate PS (post-contrast) and after PS exposure in the rat bladder from control (A-C) and WAS group (D-F). To derive the functional meaning of a slight increase in the signal intensity of WAS bladder wall, color coded, voxel-wise $\mathrm{T}_{1}$ maps with volumetric coverage were constructed from the mono exponential fit of signal in $\mathrm{T}_{1}$ weighted images acquired at six different TR values of 400-10,000 ms using aRARE factor of 2 and signal averages of 2. Just as the traditional histochemical stains reveal the biochemical differences between tissue layers, the color-coded $\mathrm{T}_{1}$ maps with the resolution of $50 \mu \mathrm{m}$ for each pixel after zero filling displays the three individual layers of mucosa (U), lamina propria (L) and detrusor (D) in bladder wall differentiated by the physical parameter of $\mathrm{T}_{1}$ relaxation time mapped to voxels sized $0.1 \times 0.1 \times 0.7 \mathrm{~mm}^{3}$. The relative ingress of extracellular $\mathrm{T}_{1}$ shortening agent, Gadobutrol in the post-contrast $\mathrm{T}_{1}$ maps expanded the spatial separation between pixels displaying the layers of $U$ and L (Eii vs Bii) and predicted an expansion of extracellular space in $\mathrm{L}$ of WAS group compared to controls. The spatial separation between $\mathrm{U}$ and $\mathrm{L}$ of WAS group increased further after a brief PS exposure (Fii vs Cii).

tion rate of water $\left(\mathrm{R} 1=1 / \mathrm{T}_{1}\right)$ is linearly related to the Gadobutrol concentration ${ }^{21,23,29}$, Gadobutrol permeability can be computed from the pre-contrast and post-contrast $\mathrm{T}_{1}$ maps using the Eq. (1)

$$
\Delta\left(\frac{1}{T 1}\right)=\frac{1}{T 1(\text { post })}-\frac{1}{T 1(\text { pre })}=r 1[G d]
$$

where [Gd] is the unknown Gadobutrol concentration, $r_{1}$ is the relaxivity of Gadobutrol (measured $3.58 \mathrm{mM}^{-1} \mathrm{~s}^{-1}$ at $9.4 \mathrm{~T}$, Fig. $2 \mathrm{~A}$ ), and 1/T1 (pre) and 1/T1 (post) are the pre- and post-contrast spin-lattice relaxation rates derived from pre-contrast and post-contrast $\mathrm{T}_{1}$ relaxation time of respective groups (Fig. $2 \mathrm{~B}$ ).

With the help of Eq. (1), we calculated significantly higher [Gd] in the mucosa (U) of WAS group compared to controls (Fig. 2C). Using the threshold of $\Delta \mathrm{T}_{1}>1500 \mathrm{~ms}$, deeper Gd penetration was measured in the WAS group $152.04 \pm 17.8 \mu \mathrm{m}$ vs $106.9 \pm 5.81 \mu \mathrm{m}$ in controls and greater still after PS exposure $193.92 \pm 16.7 \mu \mathrm{m}$ vs $125.55 \pm 9.72 \mu \mathrm{m}$ (Fig. 2D), PS is well known to evoke urothelial denudation ${ }^{25}$.

Validation of MRI permeability assay. The robustness of MRI to assay the bladder permeability of WAS model is evident from the differential impact of PS exposure on the mucosal permeability of control and WAS groups (Fig. 2C). A significant increase in the ingress of $\mathrm{Gd}(\sim 200 \mu \mathrm{M})$ in the WAS group upon PS exposure relative to controls (Fig. 2D) suggests that the stress aggravates the innate response to mild noxious stimulation in bladder, which can be relevant in understanding the flare-up of symptoms in IC/BPS symptoms.

Histological confirmation. The visual separation of $U$ and $L$ layers predicted by the post-contrast MRI of the WAS group (Fig. 1Eii) was subsequently confirmed by the H\&E staining of animals $(n=4)$ not exposed to PS (Fig. 3A-D). Compared to the low and high magnification images of control rat bladder (Fig. 3A,C) the images of WAS group without PS exposure (Fig. 3B,D) exhibited a thinned mucosa (U) with a decrease in the number of cell layers.

The extent of edema in WAS group as a consequence of inflammation led to a physical expansion of (L) layer as visible in Fig. 3D indicating that compared to controls, bladder wall thickening in WAS group occurs in conjunction with the multiple foci of mononuclear inflammatory cells $\left(^{*}\right)$, and venous congestion $(\nabla)$ and elongated 
A

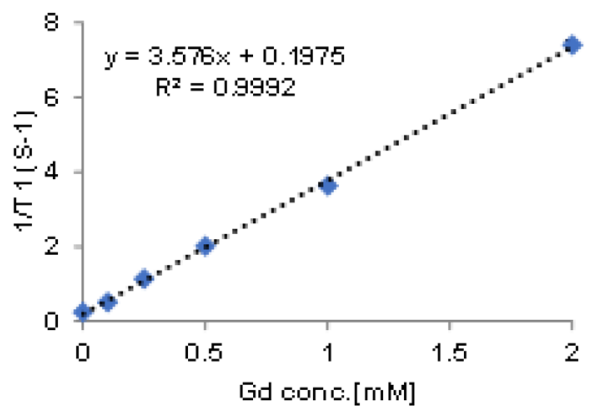

B

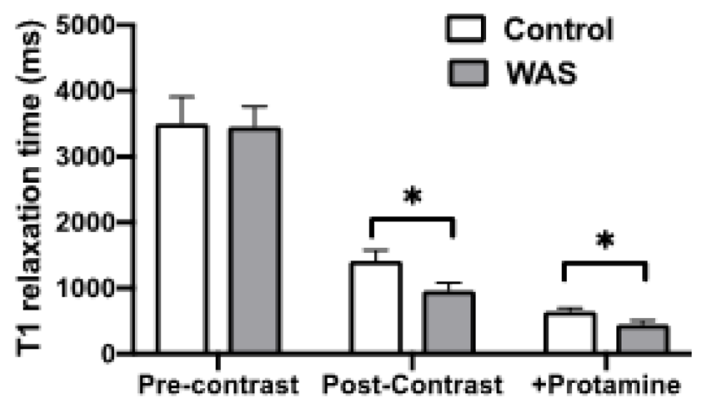

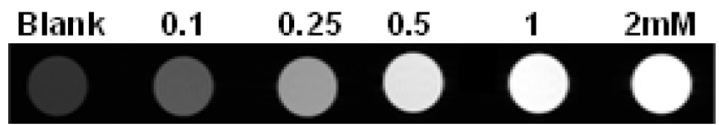
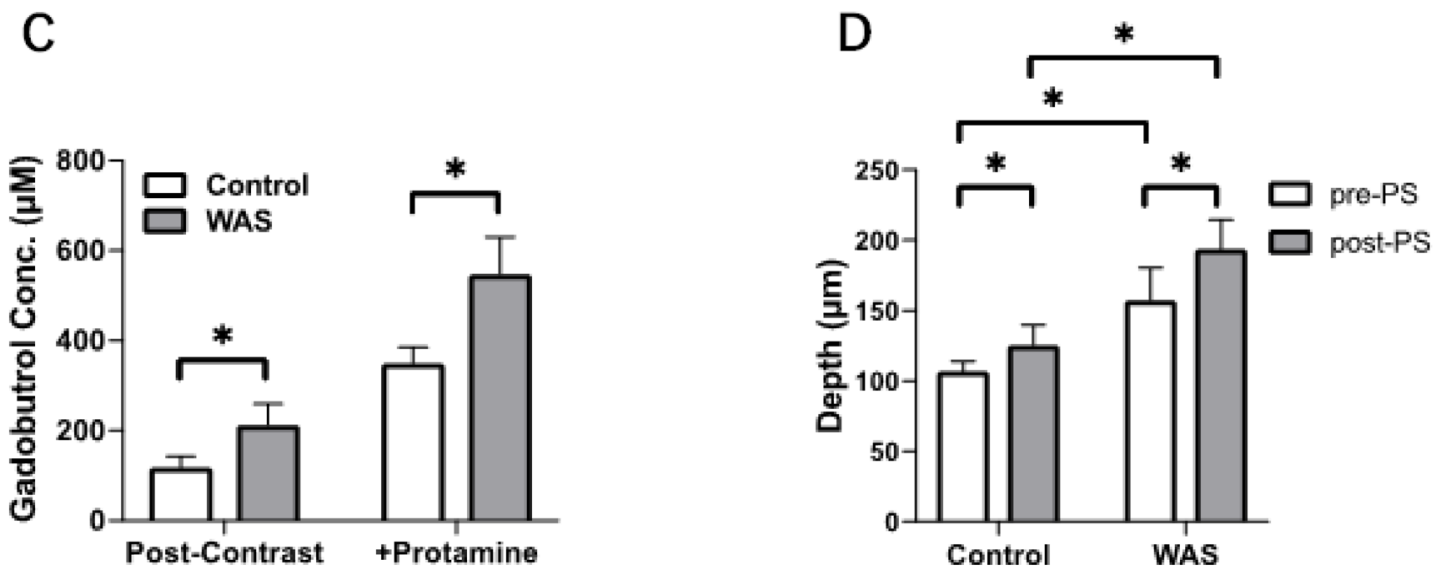

Figure 2. Gadobutrol Permeability Derived from Voxel-wise $T_{1}$ mapping: (A) To derive the Gadobutrol concentration $[\mathrm{Gd}]$ in the bladder mucosa using the Eq. (1), we first estimated the $\mathrm{T}_{1}$ relaxation rate constant or relaxivity $\left(r_{1}\right)$ of Gadobutrol at $9.4 \mathrm{~T}$ by the linear fitting of the reciprocal of the water $\mathrm{T}_{1}$ relaxation time or $\mathrm{T}_{1}$ water relaxation rate $\left(1 / \mathrm{T}_{1}\right)$ measured in the serial two fold dilutions of $[\mathrm{Gd}] 2 \mathrm{mM}$ at $37^{\circ} \mathrm{C}$. Linear fitting generated the regression equation: $y=3.576 x+0.1975$ with the coefficient of determination $\left(R^{2}\right)$ of 0.999 and $r_{1}$ value of $3.576 \mathrm{~L} / \mathrm{mmol} / \mathrm{s}$ for Gadobutrol at $9.4 \mathrm{~T}$. In contrast to the linear relationship between $\mathrm{T}_{1}$ water relaxation rate of the phosphate buffered saline (PBS) vial (blank) supplemented with ascending concentrations of Gadobutrol from 0.1-2 mM [Gd], the non-linearity between [Gd] and signal intensity is illustrated by the doubling of [Gd] from 1 to $2 \mathrm{mM}$ producing only a minor change in the signal intensity of $\mathrm{T}_{1}$ weighted images acquired at TR/TE of 640/14 ms. (B) Compared to control group, the post-contrast $\mathrm{T}_{1}$ relaxation time computed from 20 pixels in ROI of mucosa ${ }^{\star}$ was significantly shorter in WAS group ${ }^{*} p<0.005$, two-way ANOVA followed by Sidak's test) and $\mathrm{T}_{1}$ relaxation time was further shortened upon PS exposure $(0.5 \mathrm{~mL}$ of $1 \% \mathrm{w} / \mathrm{v})$ for $30 \mathrm{~min}$. (C) Higher mucosal permeability in WAS group is indexed by a significantly higher ingress of Gadobutrol derived from the non-invasive, quantitative measurement of increased $\mathrm{T}_{1}$ water relaxation rates $(\mathrm{n}=5$; ${ }^{*} p<0.001$, two-way ANOVA and Sidak's test) which is doubled from the respective pre-PS levels by PS exposure in both groups. (D) Bladder wall depth of instilled Gadobutrol penetration was evaluated by measuring the spatial separation between $U$ and $L$ on post-contrast and post-protamine $T_{1}$ maps. The penetration depth of Gadobutrol after PS in WAS group was significantly larger than in the control group ${ }^{\star} p<0.05$. All values are expressed as Mean \pm SD.

blood vessels (telangiectasia). The histology of bladder sections from control group was conspicuous by the absence of inflammatory cells. The median score of WAS group was significantly elevated compared to the control group with the statistical significance of non-normal data analyzed by Mann-Whitney test $(p<0.0001$; Fig. 3E).

Real-time PCR. The ZO-1 mRNA was downregulated in the bladder mucosa of WAS group compared to controls $(0.50 \pm 0.15$ vs $1.00 \pm 0.28, p<0.05)$ (Fig. $3 F)$.

\section{Discussion}

To bring a paradigm shift in the permeability assay of bladder mucosa, we report on the measurement of bladder wall $\mathrm{T}_{1}$ relaxivity for indexing the innate host response to chronic psychological stress and PS exposure. Since bladder wall $\mathrm{T}_{1}$ relaxivity is linearly related to the paracellular entry of $[\mathrm{Gd}]$ into the extracellular space of 


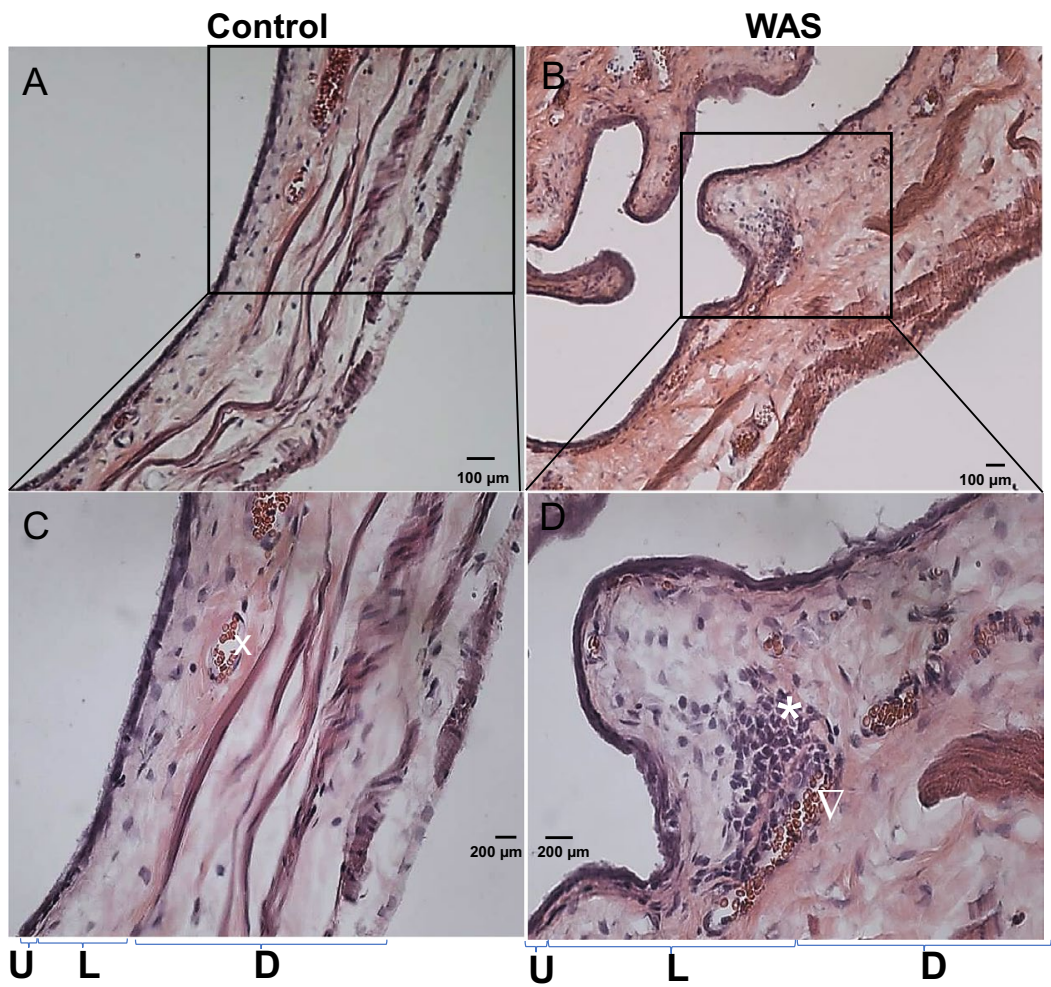

E

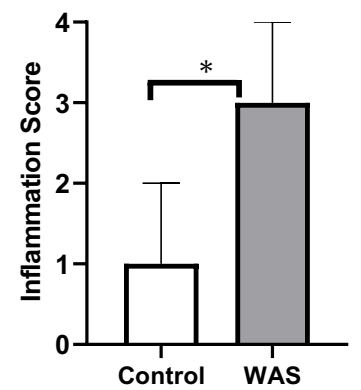

$F$

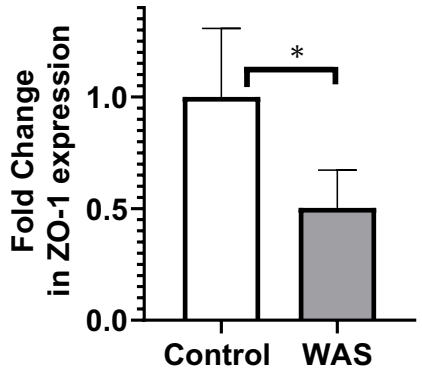

Figure 3. MRI successfully visualizes the sandwich of lamina propria (L) layer between the tissue layers of mucosa (U) and detrusor (D) and spatial separation of layers on MRI predicted the histological differences noted on H\&E staining of rat bladder harvested prior to PS exposure from both groups. Compared to the histology of control rats (A-C), tissue sections from WAS group (B-D) exhibited a relative thinning of mucosa $(\mathrm{U})$ with the decrease in the number of cell layers, expansion of lamina propria (L) from edema as evident from the relative expansion of $\mathrm{L}$ and D layers of WAS group compared to the control group (bladder wall thickening), focal areas of mononuclear inflammatory cells $\left({ }^{\star}\right)$, venous congestion and elongated blood vessels (telangiectasia $\nabla)$ together supporting a prominent role for inflammation in WAS group even without PS exposure (D). (E) A significant elevation in the median semi-quantitative score of inflammation in WAS group relative to the control group (plot of median and interquartile range as error bars, $n=4,{ }^{\star} p<0.0001$, Mann-Whitney Test) reflects the conspicuous absence of inflammatory cells in control group $(\mathbf{A}-\mathbf{C})$, a circular shape $(\mathrm{x})$ instead of elongated blood vessels and mucosa with multiple layers and thinner bladder wall signifying a relative absence of edema compared to WAS group (B-D). (F) WAS exposure evokes barrier dysfunction per se even in the absence of PS exposure as evident from histological analysis and the thinning of mucosal layers is corroborated by the downregulation of tight junction protein ZO-1 in the mucosa of WAS group. Values are Mean \pm SD; ${ }^{\star} p<0.05$, unpaired $\mathrm{t}$ test.

bladder wall either across the tight junction of capillaries after intravenous injection of Gadobutrol or its analog $)^{24}$ or across the tight junction complex of umbrella cells after instillation (as in irradiated mouse bladder ${ }^{29}$ ), we hypothesized that the quantitative $\mathrm{T}_{1}$ mapping of rat bladder can serve as a robust index for compromised bladder permeability of rat exposed to WAS ${ }^{13}$. A minimally invasive MRI based permeability assay can provide global information of bladder permeability and avoid the site-selection bias of biopsy $y^{2,45,12}$ or the complexity and errors intrinsic to biochemical assays ${ }^{10,16}$.

It is well known that the value of any physical parameter depends on the externally applied fields: e.g., just as the weight of an object increases in the higher gravitational field of Earth vs Moon, $\mathrm{T}_{1}$ relaxation time of human bladder also ascends from $0.765 \mathrm{~s}, 0.923 \mathrm{~s}$ to $1.544 \mathrm{~s}$ with the ascending magnetic field strength of $0.35 \mathrm{~T}^{34}$, $1.5 \mathrm{~T}^{24}$ and $3 \mathrm{~T}^{35}$. Indeed, the histological changes noted on the harvested rat bladder with the chemical binding of histochemical stains were predicted by the voxel-wise $\mathrm{T}_{1}$ mapping and a deeper Gadobutrol diffusion reflected an expansion of extracellular space ${ }^{23}$ in lamina propria of the WAS group, subsequently confirmed by histology. We inferred that the significantly higher ingress of [Gd] in the WAS group is in concert with the histologically confirmed thinning of the urothelium and tight junction protein, ZO-1 downregulation in separated mucosa. The functional(MRI), histologic and molecular evidence for a barrier dysfunction in WAS is corroborated by several reports on barrier dysfunction ${ }^{6-8}$ including the dilatation of intercellular spaces in the urothelium of female WAS rat ${ }^{13}$ and the report of increased shedding of urothelium in female stressed rats ${ }^{9}$. When our findings are taken together with the reported downregulation of ZO-1 and E-cadherin ${ }^{5,12}$ in the bladder biopsy of BPS/ IC patients ${ }^{5,10,11}$, we inferred that the exposure to acute or chronic psychological stress can precipitate an acute rise in bladder permeability which can exacerbate flare-up ${ }^{36}$ and/or worsen BPS/IC symptoms ${ }^{1}$. 
It is well established that PS exposure increases the bladder permeability of rodent ${ }^{25-27}$ and of human bladder ${ }^{16}$ and therefore the doubling of respective Gadobutrol ingress after PS exposure from pre- PS exposure levels in both control and WAS groups validates our MRI based mucosal permeability assay. After PS exposure, the Gadobutrol levels in bladder of WAS group were computed to be $\sim 0.5 \mathrm{mM}$ comparable to the maximum plasma levels measured $2 \mathrm{~min}$ after the intravenous injection of Gadobutrol ${ }^{37,38}$. Furthermore, the doubling of instilled Gadobutrol ingress mirrors the doubling of radiolabeled urea ingress into rabbit ${ }^{27}$ and human bladder ${ }^{16}$ after PS exposure. Moreover, the mucosal permeability barrier of live animal is critically dependent upon the oxygenation via capillary perfusion ${ }^{39,40}$ as illustrated by the ten-fold uptake of radiolabeled urea after PS exposure ex vivo ${ }^{26}$ compared to just two-fold increase in vivo ${ }^{27}$. Infact, the differences in bladder mucosal permeability ex vivo ${ }^{26}$ and in vivo ${ }^{27}$ could be easily assessed by the percentage of non-viable cells ex vivo ${ }^{20,41}$.

Since mucosal concentration build up via diffusion tends to quickly reach an equilibrium with the luminal concentration of probe, the mucosal concentration of instilled probe was found to be largely independent of the instillation period in prior computer simulation ${ }^{42}$ of distributed model of intravesical pharmacokinetics as well as in empirical studies ${ }^{43}$. This occurs because the instilled drug diffusing into capillary perfused bladder wall ${ }^{44}$ gets cleared away by the venous blood to erect a downhill concentration gradient for sustaining the paracellular diffusion into mucosa and obviate any concentration buildup in the mucosa as observed ex vivo ${ }^{26}$. Therefore, the lack of any additional rise in the [Gd] ingress with the increase in the instillation period of CM for repeat $\mathrm{T}_{1}$ mapping after PS exposure demonstrates the compliance of [Gd] ingress with the distributed model for intravesical pharmacokinetics. The extensive plasma uptake $e^{16,27}$ of the instilled radiolabeled urea in IC/BPS patients ${ }^{10}$ through a first order process validated the distributed model for intravesical pharmacokinetics. The venous clearance of instilled Gadobutrol is argued by the venous clearance of the eight times larger inulin when instilled into rat bladder ${ }^{44}$ and therefore the venous clearance of diffused Gadobutrol not only engenders a logarithmic concentration decline across the bladder wall thickness ${ }^{29}$ but also prevent any additional rise in the mucosal concentration with the repeat instillation.

The passive, concentration dependent, paracellular diffusion of instilled Gadobutrol ${ }^{29}(604.71$ Daltons), cations $^{10}$ and of large molecular weight permeability probes such Evans blue dye ( 961 Daltons $)^{17,45}$ into bladder mucosa led us to infer that the compromised mucosal barrier increases the penetration of irritants from urine for inciting the underlying afferent nerves and drive the pain and LUTS of IC/BPS patients. Thus, a significant rise in [Gd] ingress after PS exposure in the WAS group can potentially replicate the flare up of BPS/IC symptoms ${ }^{46}$ in a non-infective rodent model without relying on bacterial products.

Instead of instilling permeability probe to $80 \%$ of functional capacity in controls and BPS/IC patients ${ }^{15}$, we choose to use a constant instilled volume of $0.3 \mathrm{~mL}$ for $\mathrm{CM}$ in both groups to exclude the role of bladder distension in observed permeability differences. Moreover, $0.3 \mathrm{~mL}$ is $<50 \%$ of the average nocturnal bladder capacity of awake control rats and much lower than the threshold of $90 \%$ anaesthetized bladder capacity required for inducing any alterations in the rabbit mucosal permeability ${ }^{20}$. While WAS model recapitulates the correlation of smaller bladder capacity with the bladder wall inflammation of BPS/IC patients ${ }^{4}$, we failed to detect any increase in the urinary frequency presumably due to variable urine production during night-time or due to a decrease in total urine volume of WAS group. The psychological stress evoked by unpredictable stressors is known to affect the endocrine release of corticosterone and adrenaline, which can cause colon motility, polydipsia and sleep disturbances ${ }^{47}$. Thus, the higher number of fecal pellets measured during $1 \mathrm{~h}$ WAS exposure supports our claim of psychological stress playing a critical role in this model, but the diurnal and nocturnal variation in water intake of the WAS group must be clarified in future studies. In addition, the effect of WAS on sleep cycle needs to be addressed for fully comprehending the effect of WAS on mucosal hyperpermeability.

Since bladder permeability is a key pathophysiological mechanism, at least in a subset of BPS/IC patients $s^{1-5,10,11,16}$, our clinically viable, pain free approach of $\mathrm{T}_{1}$ mapping together with a minimally invasive, instillation of CM at constant volume holds relevance in potential phenotyping of BPS/IC patients into bladdercentric or extra-bladder phenotypes ${ }^{4}$. Instead of measuring the blood levels of instilled probes ${ }^{15}$ or urine levels ${ }^{16,27}$ to indirectly determine the levels of instilled probe taken up by bladder mucosa, MRI allows a direct, non-invasive measurement of probe levels in bladder mucosa and excludes the impact of large interindividual variations in the volume of distribution parameter ${ }^{48}$. It is likely that the failed clinical translation ${ }^{15}$ of instilled radiolabeled permeability probe levels in blood may be linked to the compromised mucosal perfusion of IC/BPS patients $s^{39}$. An eight fold elevation of instilled salicylate in feline models of $\mathrm{IC}^{49}$ and a two-fold elevation of a radiolabeled probe ( $\sim 4$ times the molecular mass of salicylate) after acute bladder injury ( akin to PS exposure) in rabbits ${ }^{15}$ failed to translate in IC/BPS patients ${ }^{15}$ because of the differences in mucosal perfusion of two cohorts ${ }^{39}$. The compromised mucosal perfusion may be a two-edged sword which can not only aggravate urothelial hyperpermeability but also hinder in the venous clearance of a diffused permeability probe before it can be measured in isolated blood aliquots. Thus, the use of intravesical contrast MRI allows us to better understand the mechanistic relationship between psychological stress and bladder dysfunction for advancing the understanding of the phenotype-specific BPS/IC pathology.

\section{Conclusion}

The findings highlight the translational relevance of a physical assay for bladder permeability using a validated model of psychologically stressed rodents together with PS exposure to mimic the flare up of BPS/IC patients. $\mathrm{T}_{1}$ weighted MRI with a minimally invasive procedure of $C M$ instillation can evaluate the bladder mucosal permeability to advance the diagnosis and the clinical care of BPS/IC patients. 
Received: 18 February 2021; Accepted: 26 August 2021

Published online: 30 September 2021

\section{References}

1. Sutcliffe, S. et al. A longitudinal analysis of urological chronic pelvic pain syndrome flares in the Multidisciplinary Approach to the Study of Chronic Pelvic Pain (MAPP) Research Network. BJU Int. 124, 522-531. https://doi.org/10.1111/bju.14783 (2019).

2. Erickson, D. R. et al. Do the National Institute of Diabetes and Digestive and Kidney Diseases cystoscopic criteria associate with other clinical and objective features of interstitial cystitis?. J. Urol. 173, 93-97. https://doi.org/10.1097/01.ju.0000146466.71311. $\mathrm{ab}(2005)$.

3. Kim, S. H., Kim, T. B., Kim, S. W. \& Oh, S. J. Urodynamic findings of the painful bladder syndrome/interstitial cystitis: a comparison with idiopathic overactive bladder. J. Urol. 181, 2550-2554. https://doi.org/10.1016/j.juro.2009.01.106 (2009).

4. Schachar, J. S. et al. Histological evidence supports low anesthetic bladder capacity as a marker of a bladder-centric disease subtype in interstitial cystitis/bladder pain syndrome. Int. Urogynecol. J. 30, 1863-1870. https://doi.org/10.1007/s00192-019-04038-0 (2019).

5. Shie, J. H. \& Kuo, H. C. Higher levels of cell apoptosis and abnormal E-cadherin expression in the urothelium are associated with inflammation in patients with interstitial cystitis/painful bladder syndrome. BJU Int. 108, E136-141. https://doi.org/10.1111/j. 1464-410X.2010.09911.x (2011).

6. Bazi, T. et al. A modulating effect of epigallocatechin gallate (EGCG), a tea catechin, on the bladder of rats exposed to water avoidance stress. Neurourol. Urodyn. 32, 287-292. https://doi.org/10.1002/nau.22288 (2013).

7. Saglam, B. et al. An aqueous garlic extract alleviates water avoidance stress-induced degeneration of the urinary bladder. BJU Int. 98, 1250-1254. https://doi.org/10.1111/j.1464-410X.2006.06511.x (2006).

8. Zeybek, A. et al. Taurine ameliorates stress-induced degeneration of the urinary bladder. Acta Histochem. 109, 208-214. https:// doi.org/10.1016/j.acthis.2006.12.001 (2007).

9. Veranic, P. \& Jezernik, K. Succession of events in desquamation of superficial urothelial cells as a response to stress induced by prolonged constant illumination. Tissue Cell 33, 280-285. https://doi.org/10.1054/tice.2001.0175 (2001).

10. Eldrup, J., Thorup, J., Nielsen, S. L., Hald, T. \& Hainau, B. Permeability and ultrastructure of human bladder epithelium. Br. J. Urol. 55, 488-492. https://doi.org/10.1111/j.1464-410x.1983.tb03354.x (1983).

11. Jhang, J. F. et al. Electron microscopic characteristics of interstitial cystitis/bladder pain syndrome and their association with clinical condition. PLoS ONE 13, e0198816. https://doi.org/10.1371/journal.pone.0198816 (2018).

12. Liu, H. T., Shie, J. H., Chen, S. H., Wang, Y. S. \& Kuo, H. C. Differences in mast cell infiltration, E-cadherin, and zonula occludens-1 expression between patients with overactive bladder and interstitial cystitis/bladder pain syndrome. Urology 80(225), e213-228. https://doi.org/10.1016/j.urology.2012.01.047 (2012).

13. Cetinel, S., Ercan, F., Cikler, E., Contuk, G. \& Sener, G. Protective effect of melatonin on water avoidance stress induced degeneration of the bladder. J. Urol. 173, 267-270. https://doi.org/10.1097/01.ju.0000145891.35810.56 (2005).

14. Wang, Z. et al. Effects of water avoidance stress on peripheral and central responses during bladder filling in the rat: A multidisciplinary approach to the study of urologic chronic pelvic pain syndrome (MAPP) research network study. PLoS ONE 12, e0182976. https://doi.org/10.1371/journal.pone.0182976 (2017).

15. Chelsky, M. J. et al. Bladder permeability in interstitial cystitis is similar to that of normal volunteers: direct measurement by transvesical absorption of 99mtechnetium-diethylenetriaminepentaacetic acid. J. Urol. 151, 346-349. https://doi.org/10.1016/ s0022-5347(17)34945-5 (1994).

16. Lilly, J. D. \& Parsons, C. L. Bladder surface glycosaminoglycans is a human epithelial permeability barrier. Surg. Gynecol. Obstet. 171, 493-496 (1990).

17. Homan, T. et al. Novel mouse model of chronic inflammatory and overactive bladder by a single intravesical injection of hydrogen peroxide. J. Pharmacol. Sci. 121, 327-337. https://doi.org/10.1254/jphs.12265fp (2013).

18. Leppilahti, M., Kallioinen, M. \& Tammela, T. L. Duration of increased mucosal permeability of the urinary bladder after acute overdistension: an experimental study in rats. Urol. Res. 27, 272-276. https://doi.org/10.1007/s002400050122 (1999).

19. Hass, M. A., Nichol, P., Lee, L. \& Levin, R. M. Estrogen modulates permeability and prostaglandin levels in the rabbit urinary bladder. Prostaglandins Leukot. Essent. Fatty Acids 80, 125-129. https://doi.org/10.1016/j.plefa.2008.11.010 (2009).

20. Levin, R. et al. Trypan blue as an indicator of urothelial integrity. Neurourol. Urodyn. 9, 269-279 (1990).

21. Taheri, S., Shah, N. J. \& Rosenberg, G. A. Analysis of pharmacokinetics of Gd-DTPA for dynamic contrast-enhanced magnetic resonance imaging. Magn. Reson. Imaging 34, 1034-1040. https://doi.org/10.1016/j.mri.2016.04.014 (2016).

22. Barnes, S. R. et al. Optimal acquisition and modeling parameters for accurate assessment of low Ktrans blood-brain barrier permeability using dynamic contrast-enhanced MRI. Magn. Reson. Med. 75, 1967-1977. https://doi.org/10.1002/mrm.25793 (2016).

23. Steward, M. C., Seo, Y., Rawlings, J. M. \& Case, R. M. Water permeability of acinar cell membranes in the isolated perfused rabbit mandibular salivary gland. J. Physiol. 431, 571-583. https://doi.org/10.1113/jphysiol.1990.sp018348 (1990).

24. Kanazawa, Y., Miyati, T. \& Sato, O. Hemodynamic analysis of bladder tumors using T1-dynamic contrast-enhanced fast spin-echo MRI. Eur. J. Radiol. 81, 1682-1687. https://doi.org/10.1016/j.ejrad.2011.04.013 (2012).

25. Tyagi, P. et al. Novel contrast mixture improves bladder wall contrast for visualizing bladder injury. Am. J. Physiol. Renal. Physiol. 313, F155-F162. https://doi.org/10.1152/ajprenal.00609.2016 (2017).

26. Lavelle, J. et al. Bladder permeability barrier: recovery from selective injury of surface epithelial cells. Am. J. Physiol. Renal. Physiol. 283, F242-253. https://doi.org/10.1152/ajprenal.00307.2001 (2002).

27. Nickel, J. C., Downey, J., Morales, A., Emerson, L. \& Clark, J. Relative efficacy of various exogenous glycosaminoglycans in providing a bladder surface permeability barrier. J. Urol. 160, 612-614 (1998).

28. Matos, R. et al. The water avoidance stress induces bladder pain due to a prolonged alpha1 A adrenoceptor stimulation. Naunyn. Schmiedebergs Arch. Pharmacol. 390, 839-844. https://doi.org/10.1007/s00210-017-1384-1 (2017).

29. Singh, N. et al. Virtual measurements of paracellular permeability and chronic inflammation via color coded pixel-wise T1 mapping. Am. J. Physiol. Renal. Physiol. 319, F506-F514. https://doi.org/10.1152/ajprenal.00025.2020 (2020).

30. Funahashi, Y. et al. Bladder overactivity and afferent hyperexcitability induced by prostate-to-bladder cross-sensitization in rats with prostatic inflammation. J. Physiol. 597, 2063-2078. https://doi.org/10.1113/JP277452 (2019).

31. Mizoguchi, S. et al. The role of prostaglandin and E series prostaglandin receptor type 4 receptors in the development of bladder overactivity in a rat model of chemically induced prostatic inflammation. BJU Int. 124, 883-891. https://doi.org/10.1111/bju.14845 (2019).

32. Wada, N. et al. The effect of neutralization of nerve growth factor (NGF) on bladder and urethral dysfunction in mice with spinal cord injury. Neurourol. Urodyn. 37, 1889-1896. https://doi.org/10.1002/nau.23539 (2018).

33. Carattino, M. D. et al. Bladder filling and voiding affect umbrella cell tight junction organization and function. Am. J. Physiol. Renal. Physiol. 305, F1158-1168. https://doi.org/10.1152/ajprenal.00282.2013 (2013).

34. Fisher, M. R., Hricak, H. \& Crooks, L. E. Urinary bladder MR imaging. Part I. Normal and benign conditions. Radiology 157, 467-470. https://doi.org/10.1148/radiology.157.2.4048457 (1985).

35. Tyagi, P., Janicki, J., Moon, C. H., Kaufman, J. \& Chermansky, C. Novel contrast mixture achieves contrast resolution of human bladder wall suitable for T1 mapping: Applications in interstitial cystitis and beyond. Int. Urol. Nephrol. 50, 401-409. https://doi. org/10.1007/s11255-018-1794-0 (2018). 
36. Kaneko, G., Nishimoto, K., Ito, Y. \& Uchida, A. The combination therapy of prednisolone and tacrolimus for severe painful bladder syndrome/interstitial cystitis. Can. Urol. Assoc. J. 6, E46-49. https://doi.org/10.5489/cuaj.10134 (2012).

37. Scott, L. J. Gadobutrol: A review of its use for contrast-enhanced magnetic resonance imaging in adults and children. Clin. Drug. Investig. 33, 303-314. https://doi.org/10.1007/s40261-013-0066-0 (2013).

38. Vogler, H. et al. Pre-clinical evaluation of gadobutrol: a new, neutral, extracellular contrast agent for magnetic resonance imaging. Eur. J. Radiol. 21, 1-10. https://doi.org/10.1016/0720-048x(95)00679-k (1995).

39. Pontari, M. A., Hanno, P. M. \& Ruggieri, M. R. Comparison of bladder blood flow in patients with and without interstitial cystitis. J. Urol. 162, 330-334 (1999).

40. Chai, T. C., Russo, A., Yu, S. \& Lu, M. Mucosal signaling in the bladder. Auton. Neurosci. 200, 49-56. https://doi.org/10.1016/j. autneu.2015.08.009 (2016).

41. Di Stasi, S. M. et al. Electromotive versus passive diffusion of mitomycin C into human bladder wall: Concentration-depth profiles studies. Cancer Res. 59, 4912-4918 (1999).

42. Smith, S. G., Griffith, B. E. \& Zaharoff, D. A. Analyzing the effects of instillation volume on intravesical delivery using biphasic solute transport in a deformable geometry. Math. Med. Biol. 36, 139-156. https://doi.org/10.1093/imammb/dqy004 (2019).

43. Wientjes, M. G., Dalton, J. T., Badalament, R. A., Drago, J. R. \& Au, J. L. Bladder wall penetration of intravesical mitomycin C in dogs. Cancer Res. 51, 4347-4354 (1991).

44. Au, J. L., Dalton, J. T. \& Wientjes, M. G. Evidence of significant absorption of sodium salicylate from urinary bladder of rats. J. Pharmacol. Exp. Ther. 258, 357-364 (1991).

45. Elsen, S. et al. Evans blue-mediated white-light detection of non-muscle-invasive bladder cancer: A preclinical feasibility and safety study using a rat bladder urothelial cell carcinoma model. Mol. Clin. Oncol. 5, 678-688. https://doi.org/10.3892/mco.2016. 1043 (2016).

46. Kogan, P. et al. Sub-noxious intravesical lipopolysaccharide triggers bladder inflammation and symptom onset in a transgenic autoimmune cystitis model: a mapp network animal study. Sci. Rep. 8, 6573. https://doi.org/10.1038/s41598-018-24833-x (2018).

47. Dewasmes, G., Loos, N., Delanaud, S., Dewasmes, D. \& Ramadan, W. Pattern of rapid-eye movement sleep episode occurrence after an immobilization stress in the rat. Neurosci. Lett. 355, 17-20. https://doi.org/10.1016/j.neulet.2003.10.031 (2004).

48. Holness, J. L., Fleming, J. S., Malaroda, A. L. \& Warwick, J. M. (99m)Tc-DTPA volume of distribution, half-life and glomerular filtration rate in normal adults. Nucl. Med. Commun. 34, 1005-1014. https://doi.org/10.1097/MNM.0b013e328364aa12 (2013).

49. Gao, X., Buffington, C. A. \& Au, J. L. Effect of interstitial cystitis on drug absorption from urinary bladder. J. Pharmacol. Exp. Ther. 271, 818-823 (1994).

\section{Author contributions}

T.S.: protocol/project development, data collection or management, data analysis, manuscript writing/editing. T.H.: protocol/project development, manuscript writing/editing. L.F.: protocol/project development, data collection, manuscript writing/editing. N.S.: data collection. S.M.: data collection. M.K.: data collection. D.G.: data collection. T.O.: manuscript editing. T.M.: manuscript editing. O.I.: manuscript editing. C.C.: manuscript editing. J.K.: manuscript editing and grant award. N.Y.: protocol/project development, manuscript editing. P.T.: grant award, protocol/project development, data analysis, manuscript writing/editing.

\section{Funding}

National Institute of Diabetes and Digestive and Kidney Diseases, Grant number DK108397.

\section{Competing interests}

The authors declare no competing interests, except Jonathan Kaufmann, who is the CEO of Lipella Pharmaceuticals and an inventor of the contrast mixture used in the described study.

\section{Additional information}

Supplementary Information The online version contains supplementary material available at https://doi.org/ 10.1038/s41598-021-98504-9.

Correspondence and requests for materials should be addressed to P.T.

Reprints and permissions information is available at www.nature.com/reprints.

Publisher's note Springer Nature remains neutral with regard to jurisdictional claims in published maps and institutional affiliations.

Open Access This article is licensed under a Creative Commons Attribution 4.0 International

License, which permits use, sharing, adaptation, distribution and reproduction in any medium or format, as long as you give appropriate credit to the original author(s) and the source, provide a link to the Creative Commons licence, and indicate if changes were made. The images or other third party material in this article are included in the article's Creative Commons licence, unless indicated otherwise in a credit line to the material. If material is not included in the article's Creative Commons licence and your intended use is not permitted by statutory regulation or exceeds the permitted use, you will need to obtain permission directly from the copyright holder. To view a copy of this licence, visit http://creativecommons.org/licenses/by/4.0/.

This is a U.S. Government work and not under copyright protection in the US; foreign copyright protection may apply 2021 